\title{
Prefrontal Dysfunction in First-Degree Relatives of Schizophrenia Patients during a Stroop Task
}

\author{
Theresa M Becker', John G Kerns', Angus W MacDonald III' and Cameron S Carter, ${ }^{*}$ \\ 'Department of Psychological Sciences, University of Missouri-Columbia, Columbia, MO, USA; ${ }^{2}$ Department of Psychology, University of \\ Minnesota, Minneapolis, MN, USA; ${ }^{3}$ Departments of Psychiatry and Psychology, University of California at Davis, Davis, CA, USA
}

\begin{abstract}
Schizophrenia patients perform poorly on cognitive control tasks and exhibit dysfunction in anterior cingulate cortex (ACC) and dorsolateral prefrontal cortex (DLPFC) during task performance. The unaffected relatives of patients with schizophrenia also exhibit poor cognitive control task performance. However, the relationship between these behavioral deficits in relatives and the integrity of ACC and DLPFC functioning is unclear. In the present study, we used the Stroop color-naming task and event-related fMRI to examine cognitive control task performance and associated neural activity in 17 unaffected relatives of schizophrenia patients and 17 demographically matched healthy controls. On the Stroop task, unaffected relatives exhibited intact post-conflict-related performance adjustments. $\mathrm{fMRI}$ data revealed that unaffected relatives exhibited reduced activity in DLPFC but they exhibited intact activity in ACC. These results suggest that DLPFC dysfunction may be related to the genetic risk for schizophrenia as both patients and their unaffected relatives show reduced activity in this region. In contrast, the current results suggest that ACC dysfunction in people with schizophrenia may reflect processes specific to the illness itself.

Neuropsychopharmacology (2008) 33, 2619-2625; doi:I0.1038/sj.npp. I30 I673; published online 23 January 2008
\end{abstract}

Keywords: schizophrenia; relatives; endophenotype; dorsolateral prefrontal cortex; anterior cingulate; cognitive control

\section{INTRODUCTION}

People with schizophrenia exhibit impaired performance on cognitive control tasks (Cohen and Servan-Schreiber, 1992). Cognitive control refers to a set of processes involved in carrying out goal-directed behavior in the face of conflict (eg, from a highly automatic or prepotent response; Miller and Cohen, 2001; Rougier et al, 2005). Cognitive control is supported by a distributed neural network that includes the anterior cingulate cortex (ACC) and dorsolateral prefrontal cortex (DLPFC; Cohen et al, 2000). A broadly supported view of ACC function is that it monitors response conflict (ie the simultaneous activation of multiple responses) and that ACC conflict monitoring results in the recruitment of DLPFC to reduce conflict (Botvinick et al, 2001). One broadly supported view of DLPFC function is that it provides a contextually appropriate or task-relevant biasing influence to posterior brain regions, thereby reducing conflict (Miller and Cohen, 2001). Hence, both ACC and DLPFC appear to be involved in cognitive control. In addition, previous research has found evidence of both ACC and DLPFC dysfunction in people with schizophrenia

*Correspondence: Dr CS Carter, UC Davis Imaging Research Center, $4701 \times$ Street, Sacramento, CA 95817, USA, Tel: + 19167347783 , Fax: + I 916734 8750, E-mail: cameron.carter@ucdmc.ucdavis.edu Received 14 August 2007; revised 16 November 2007; accepted 16 December 2007
(Barch, 2006; Carter et al, 2001; MacDonald and Carter, 2003).

The goal of the current research was to examine whether increased liability for schizophrenia was also associated with ACC and/or DLPFC dysfunction. One way to examine the nature of the genetic liability for schizophrenia is to examine schizophrenia patients' healthy, first-degree relatives (Gottesman and Gould, 2003). One advantage to research on people with increased risk of schizophrenia is that it removes some confounds present in schizophrenia research such as medication and chronicity effects. In addition, this type of research helps to disentangle state and trait markers of the illness. The current research examined whether ACC or DLPFC dysfunction would be observed in first-degree relatives of people with schizophrenia.

A few previous studies have examined whether the unexpressed genetic liability for schizophrenia is associated with either DLPFC or ACC dysfunction while performing cognitive control tasks. Research conducted thus far has consistently found evidence of DLPFC dysfunction in the unaffected first-degree relatives of schizophrenia patients. These studies have reported both increased DLPFC activity (Callicott et al, 2003a; Thermenos et al, 2004) and decreased DLPFC activity (Keshevan et al, 2002; MacDonald et al, 2006) in relatives. Differences in the amount of DLPFC activity exhibited across studies may reflect a lack of physiological efficiency in the prefrontal cortex (eg, greater DLPFC activity at lower memory loads in relatives; Callicott 
et al, 2000, 2003b). Furthermore, it has been suggested that hypo- and hyperfrontality may depend on a number of factors such as level of working memory load and working memory task parameters (eg, processes required and cognitive domain; Manoach, 2003). fMRI task design may be another factor that contributes to the elicitation of different levels of DLPFC activity. For instance, the studies reporting hyperfrontality involved block designs. Eventrelated designs can examine neural activity associated with specific cognitive demands. One event-related study examining activity in relatives for specific cognitive control demands found decreased DLPFC activity in relatives (MacDonald et al, 2006). In the current study, we used an event-related design to examine whether the relatives of people with schizophrenia exhibited decreased DLPFC activity during cognitive control demands. Similar to the study conducted by MacDonald et al (2006), the current study involved a high level of cognitive control demands.

For ACC activity, two previous studies found greater ACC activity in relatives while performing working memory tasks (Callicott et al, 2003a; Thermenos et al, 2004). Additionally, one study found robust ACC activity in relatives and no differences in ACC activity (MacDonald et al, 2006). In contrast, one study has found decreased ACC activity (Whalley et al, 2004). However, none of these studies used tasks that are known to strongly involve response conflict and to strongly activate ACC and elicit ACC-related behavioral conflict adaptation effects. In the current study, participants performed the Stroop task, which involves high response conflict and strongly activates ACC in controls (both for response conflict and for errors; Carter et al, 2000).

\section{MATERIALS AND METHODS}

\section{Participants}

Participants were 22 unaffected first-degree relatives of schizophrenia patients (ie, 16 siblings, 5 offspring, and 1 mother) and 23 demographically matched controls (Table 1). Diagnoses for schizophrenia probands were made using the Structural Clinical Interview for the DSMIV (SCID; First et al, 1996). All controls and relatives were screened for Axis I disorders and substance abuse using the SCID-NP (Spitzer et al, 1990). The relatives were also administered the Structural Interview for DSM-III Personality Disorders (Pfohl et al, 1982) to assess for any schizophrenia-spectrum personality disorders. One relative

Table I Demographic Information for Relatives and Controls

\begin{tabular}{lccr}
\hline Demographic variable & Relatives & Controls & t-Score $\boldsymbol{\chi}^{\mathbf{2}}$ \\
\hline Male/female & $6 / 11$ & $10 / 7$ & 1.91 \\
C/AA & $10 / 7$ & $13 / 4$ & 1.22 \\
Age (years) & $33.3(10.8)$ & $32.7(7.8)$ & -0.18 \\
Education & $14.9(2.8)$ & $15.7(1.9)$ & 1.04 \\
Parental education & $13.9(2.5)$ & $13.4(2.2)$ & -0.61 \\
\hline
\end{tabular}

AA, African American; C, Caucasian.

All tests were nonsignificant; means and standard deviations reported. met criteria for schizotypal personality disorder. Two controls and three relatives were removed because of problems with their functional data (eg, excessive movement). Four controls and two relatives were excluded from the analysis due to inadequate behavioral data. Specifically, of these six participants, two controls and one relative did not make enough errors (ie, less than eight errors), which was necessary for the behavioral error analysis. In addition, two controls and one relative performed close to chance (ie, error rates $>60 \%$ ). Therefore, all reported analyses involved 17 controls and 17 relatives.

\section{Stroop Task}

After providing informed consent, subjects performed the Stroop color-naming task during scanning. Stimuli consisted of one of three words (RED, GREEN, BLUE) printed in one of the three colors. Trials were either congruent (eg, the word 'BLUE' written in blue ink) or incongruent (eg, the word 'BLUE' written in red ink). For all trials, participants were instructed to respond to the color of the stimulus and to ignore the word. Participants responded with a button press using the index, middle, and ring fingers on their right hand and were instructed to respond both quickly and accurately. Trials consisted of a color word for $1 \mathrm{~s}$ followed by $2 \mathrm{~s}$ of a fixation cross $(+)$. There were 3 blocks of 88 trials each. To increase conflict effects, the first and last four trials of every block were congruent, and of the remaining 80 trials within each block, $70 \%$ were congruent and 30\% were incongruent (Carter et al, 2000). To examine the in-scanner behavioral data, we analyzed three separate behavioral effects. First, we examined the Stroop effect in both relatives and controls (ie, difference in reaction times (RTs) between incongruent and congruent trials). We also analyzed two additional behavioral effects (Botvinick et al, 2001): post-conflict adjustments ((iC-cC) $+(\mathrm{cI}-\mathrm{iI})$, ie, $\mathrm{iC}=$ congruent trial preceded by an incongruent trial) and post-error adjustments (ie, difference in RT between trials after errors and trials after correct responses).

\section{Imaging Acquisition and Pre-Processing}

A $3.0 \mathrm{~T}$ General Electric scanner with a standard head coil was used to acquire all images. Three gradient echo structural scouts were used to localize the anterior and posterior commissure. A high-resolution 124-slice structural image (SPGR) was acquired as well. $\mathrm{T}^{*}$ spiral scans $\left(3.2 \mathrm{~mm}^{3}\right.$ voxels, repetition time $=1.5 \mathrm{~s}$, echo time $=18 \mathrm{~ms}$, flip angle $=70^{\circ}$ ) acquired 28 axial slices for the majority of the participants and 26 axial slices for five of the relatives and four of the controls (difference in total slice number due to a scanner upgrade).

Incremental (scan to scan) and total movements were corrected using Automatic Image Registration (Woods et al, 1998). No significant difference was found in movement between the controls and unaffected relatives (all $p$ 's $>0.28$ ). A 30-parameter nonlinear warping algorithm was used to align each participant's SPGR image to the Montreal Neurological Institute (MNI152) single-subject high-resolution anatomical reference brain (Evans et al, 1993). The parameter estimates from the nonlinear warping algorithm 
were applied to the functional $\mathrm{T} 2{ }^{*}$-weighted images to bring all participants' data into alignment with the MNI reference brain. The data were then smoothed in three dimensions using an $8 \mathrm{~mm}$ FWHM kernel to accommodate individual differences in brain morphology.

\section{Imaging Statistical Analysis}

Imaging data were analyzed with a random effects singlesubjects general linear model (GLM) using AFNI (Cox, 1996) and NIS software implemented within a fiswidgets desktop (Fissell et al, 2002). For all analyses, we ran a GLM for each participant using a canonical double gamma hemodynamic response function to obtain a parameter estimate (ie, beta value) for each covariate for each participant. We then performed $t$-tests to examine significant activity both within groups and between groups. To correct for the multiple statistical comparison problem, the statistical significance threshold for all analyses was $p<0.005$ and eight contiguous voxels, reducing the probability of a false positive to $p<0.0005$ for all regions of interest found (Forman et al, 1995). The analyses that were conducted included five covariates (incongruent, congruent, errors, no responses, and stimulus repetitions; Mayr et al, 2003). These analyses tested all voxels and produced ROIs of ACC and DLPFC conflict-related activity. The design and method of the current study using only correct trials have been previously used successfully (Kerns et al, 2004, 2005).

\section{RESULTS}

\section{Behavioral Performance}

In a 2 (current trial type: congruent $v s$ incongruent) by 2 (previous trial type: congruent $v s$ incongruent) by 2 (group: controls $v s$ relatives) analysis of variance, there was a main effect of group (ie, unaffected relatives were overall significantly slower than controls), $\mathrm{F}(1,32)=4.198, p<0.05$. In addition, there was a main effect of current trial type (ie, the Stroop effect), $\mathrm{F}(1,32)=46.017, p<0.001$ (congruent mean: 556.5, $\mathrm{SD}=126.5$; incongruent mean: 686.8, $\mathrm{SD}=224.4)$. Unaffected relatives did not significantly differ from controls in the Stroop interference effect (ie, current trial type $\times$ group interaction), $F(1,32)=1.858$, $p=0.18$, although relatives did have a numerically larger interference effect (between-group interference effect size Cohen's $d=0.48$, close to a medium effect; controls: congruent mean $=513.0 \mathrm{~ms}, \mathrm{SD}=91.5$; incongruent mean$617.1 \mathrm{~ms}, \mathrm{SD}=151.2$; relatives: congruent mean $=600.0 \mathrm{~ms}$, $\mathrm{SD}=143.5$; incongruent mean $=756.5 \mathrm{~ms}, \mathrm{SD}=265.8$ ).

In the examination of trial-to-trial adjustments in task performance, there was a significant current trial type by previous trial type interaction (ie, post-conflict adjustment effect), as there was a significant post-conflict adjustment effect, $p<0.05$. However, the current trial type by previous trial type by group interaction was not significant, $\mathrm{F}(1,32)=0.643, p<0.43$, with relatives exhibiting intact post-conflict adjustments and numerically larger adjustments than controls (Cohen's $d=-0.23$; relatives postconflict adjustments: $\mathrm{cC}$ mean $=587.8 \mathrm{~ms}, \mathrm{SD}=138.3$; cI mean $=759.2 \mathrm{~ms}, \quad S D=268.6, \quad$ iC $\quad$ mean $=616.3 \mathrm{~ms}$,
$\mathrm{SD}=154.7$; iI mean $=746.6 \mathrm{~ms}, \mathrm{SD}=264.3$ ). Furthermore, relatives did not differ from controls on amount of posterror slowing, $F(1,32)=1.447, p=0.23$, with relatives exhibiting intact post-error slowing and numerically larger post-error slowing than controls (Cohen's $d=-0.43$; relatives post-error adjustments: RT after correct mean$633.2 \mathrm{~ms}, \mathrm{SD}=171.9$; RT after incorrect mean $=674.2 \mathrm{~ms}$, $\mathrm{SD}=188.6)$.

\section{Functional Brain Activity}

As shown in Table 2, during high conflict incongruent trials, both controls and relatives exhibited activity in many regions typically activated by cognitive control demands, including inferior frontal gyrus and inferior parietal cortex. In addition, as shown in Table 2 as well as in Figure 1a and b, both controls and relatives exhibited significant conflictrelated activity in the ACC during high conflict trials (controls: BA 24; relatives: BA32). However, only controls exhibited significant left DLPFC activity, whereas relatives did not.

As shown in Table 3 as well as Figures 2 and 3, the relatives exhibited significantly less activity in the left DLPFC (BA 9) compared to controls. Furthermore, the group difference was found significant at $p<0.005$ and nine voxels, reducing the probability of a false positive to $p<0.0005$. However, there were no significant differences between groups in ACC activity, with, if anything, the

Table 2 Regions Exhibiting Significant Conflict-Related Activity in Controls and Relatives

\begin{tabular}{|c|c|c|c|c|}
\hline Region/BA & $x$ & y & $\mathbf{z}$ & Max t-score \\
\hline \multicolumn{5}{|l|}{ Controls } \\
\hline Anterior cingulate cortex, BA 24/32 & 0 & 6 & 48 & 3.90 \\
\hline Left middle frontal gyrus, BA 9 & -46 & 15 & 42 & 6.34 \\
\hline Left middle frontal gyrus, BA 44/9 & -50 & 18 & 30 & 6.34 \\
\hline Left middle frontal gyrus, BA 46 & -46 & 38 & 8 & 6.34 \\
\hline Left inferior parietal lobule, BA 40 & -43 & -52 & 42 & 4.70 \\
\hline Left inferior frontal gyrus, BA 6/44 & -40 & 2 & 31 & 6.34 \\
\hline Left inferior frontal gyrus, BA 44/4 & -43 & 14 & 21 & 6.34 \\
\hline Left inferior frontal gyrus, BA 47 & -40 & 16 & -2 & 4.32 \\
\hline Right inferior frontal gyrus, BA 47 & 49 & 19 & -8 & 5.23 \\
\hline \multicolumn{5}{|l|}{ Relatives } \\
\hline Anterior cingulate cortex, BA 32/6 & 0 & 15 & 42 & 4.17 \\
\hline Left precuneus, BA 7/19 & -21 & -67 & 37 & 4.03 \\
\hline Right cuneus, BA 18/19 & 6 & -92 & 27 & 4.16 \\
\hline Left inferior parietal lobule, BA 40 & -59 & -52 & 25 & 4.98 \\
\hline Left inferior frontal gyrus, BA 45 & -40 & 16 & -2 & 5.51 \\
\hline Left inferior frontal gyrus, BA 47 & -46 & 19 & -5 & 6.39 \\
\hline Right inferior frontal gyrus, BA 47 & 49 & 46 & -4 & 7.86 \\
\hline Right inferior frontal gyrus, BA 47 & 46 & 19 & -5 & 6.12 \\
\hline Left middle temporal gyrus, BA 21 & -62 & -39 & -2 & 5.38 \\
\hline Right superior temporal gyrus, BA 38 & 49 & 18 & -16 & 5.38 \\
\hline
\end{tabular}

Abbreviation: BA, Brodmann areas.

$x, y, z$ refer to Talaraich coordinates for peak area of activation; all activated regions $p<0.005$ and at least eight contiguous voxels. 

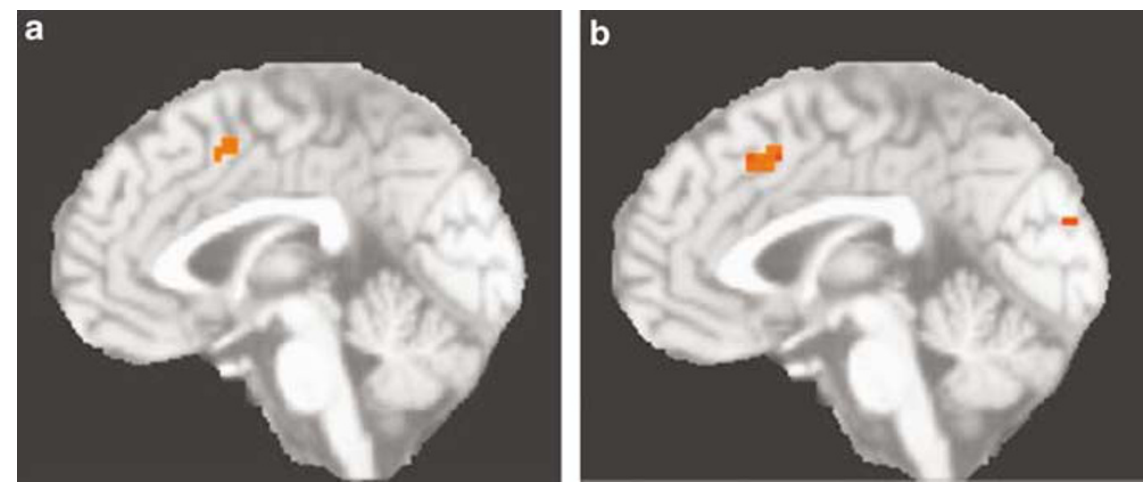

Figure I (a) Significant conflict ACC activity in controls. (b) Significant conflict ACC activity in relatives.

Table 3 Regions Exhibiting Significant between-Group Differences in Conflict-Related Activity

\begin{tabular}{lrrrr}
\hline Region/BA & x & y & z & Max t-score \\
\hline $\begin{array}{l}\text { Controls > relatives } \\
\text { Left middle frontal gyrus, BA 9 }\end{array}$ & -40 & -1 & 41 & 3.80 \\
& & & & \\
$\begin{array}{l}\text { Relatives }>\text { controls } \\
\text { Left inferior parietal lobule, BA 40 }\end{array}$ & -53 & -40 & 30 & -3.77 \\
$\quad$ Right inferior frontal gyrus, BA 10/47 & 53 & 46 & -4 & -3.28 \\
\hline
\end{tabular}

BA, Brodmann areas.

$x, y, z$ refer to Talaraich coordinates for peak area of activation; all activated regions $p<0.005$ and at least 8 contiguous voxels.

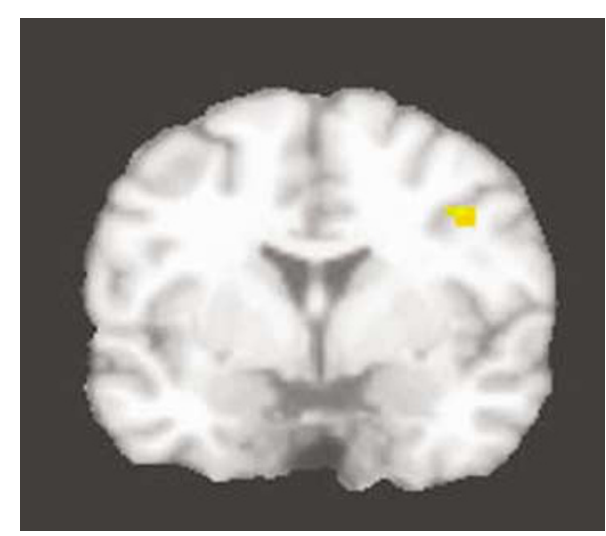

Figure 2 Group difference in left DLPFC activity with controls activating more than relatives in an overlapping region (note: the right side of the figure is the left side of the brain).

relatives exhibiting numerically but non-significantly greater activity in the ACC (Figure 3). Similarly, during error trials, both controls and relatives exhibited significant error-related activity in the ACC, and there were no significant differences between groups (controls: BA 24/32; relatives: BA 32).

There were only two other group differences found during high conflict incongruent trials. Specifically, the relatives exhibited significantly increased activity compared to

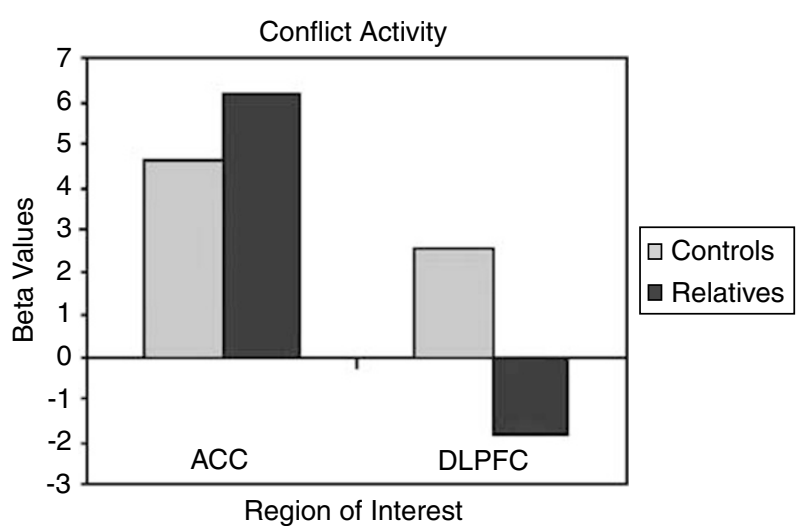

Figure 3 Beta values plotted for each region of interest in both controls and relatives.

Table 4 Correlations between Regions of Interest

\begin{tabular}{lcccc}
\hline & ACC & $\begin{array}{c}\text { Left } \\
\text { DLPFC }\end{array}$ & $\begin{array}{c}\text { Left } \\
\text { parietal }\end{array}$ & $\begin{array}{c}\text { Right inferior } \\
\text { frontal }\end{array}$ \\
\hline ACC & - & 0.41 & -0.24 & $-0.58^{*}$ \\
Left DLPFC & 0.35 & - & -0.31 & -0.28 \\
Left parietal & 0.38 & $0.44^{\dagger}$ & - & 0.40 \\
Right inferior & 0.09 & -0.11 & -0.10 & - \\
frontal & & & & \\
\hline
\end{tabular}

${ }^{\dagger} p=0.08$; * $p<0.05$; correlations for controls are above the diagonal and correlations for relatives are below the diagonal.

controls in the left inferior parietal lobule (BA 40) and in the right inferior frontal gyrus (BA 10/47; Table 3).

Given that relatives exhibited both decreased and increased activity compared to controls, exploratory analyses examined whether the relationship between activities in these brain regions differed between relatives and controls. As shown in Table 4, some of the associations between brain regions were different between relatives and controls. In an analysis comparing the size of Pearson correlations between relatives and controls, the correlation between ACC and the inferior parietal lobule was stronger for relatives than for controls at the trend level $(Z=1.71$, $p=0.09$ ). Furthermore, the correlations between ACC and 
inferior frontal gyrus $(Z=1.99, p<0.05)$ and between DLPFC and the inferior parietal lobule $(Z=2.10, p<0.05)$ were significantly stronger in relatives compared to controls. Hence, it appears that the functional relationships between these regions might be different for controls and relatives. For controls, ACC and DLPFC activities appear somewhat (but nonsignificantly) positively related. In contrast, the more control participants activated ACC and DLPFC, the less they tended to activate parietal and inferior frontal regions. In contrast, for relatives, the more they activated ACC and DLPFC, the more they also activated a parietal region.

\section{DISCUSSION}

The current study is the first to use the Stroop task to examine the neural correlates of cognitive control demands in unaffected first-degree relatives of people with schizophrenia. In this study, relatives exhibited a significant reduction in left DLPFC (BA9) during conflict trials compared to controls. Hence, the current study is the first to report decreased DLPFC activity in a large sample size of unaffected relatives using a task involving robust response conflict. In addition, not only did the relatives differ significantly from controls, but also failed to exhibit significant conflict-related DLPFC activity in this study. Overall, the current study suggests that DLPFC dysfunction occurs in first-degree relatives as well as individuals with schizophrenia.

In contrast to decreased DLPFC activity, relatives exhibited significant conflict and error-related ACC activity, and relatives did not differ from control participants in amount of ACC activity. Moreover, relatives exhibited intact post-conflict and post-error behavioral adjustments, which to our knowledge were examined for the first time in relatives in this study. Importantly, the Stroop task involves a high amount of response conflict and has been found to strongly activate the ACC in controls (Carter et al, 2000). Hence, if relatives of people with schizophrenia have ACC dysfunction, it would be expected to be evident on the Stroop task. However, the current study with an adequate sample size found no evidence of ACC dysfunction in relatives. Overall, the current study suggests that ACC activity may be intact in first-degree relatives.

The current results suggest that DLPFC but not ACC dysfunction may be present in first-degree relatives of schizophrenia patients. Importantly, evidence of DLPFC dysfunction in the current study cannot be easily accounted for by failure to detect brain activity in relatives. In the current study, relatives exhibited increased activity in some regions compared to controls (ie, the inferior parietal lobule and inferior frontal gyrus), which might reflect a compensatory mechanism in the face of decreased DLPFC activity (Barch, 2005). Furthermore, for relatives, the more they activate the DLPFC, the more they activate a parietal region, but this pattern of results is not present in controls. Similarly, Tan et al (2006) found greater activation in the inferior frontal gyrus compared to the DLPFC in response to working memory load; however, in that study, a stronger correlation was found between the inferior frontal and parietal regions than between the DLPFC and parietal regions. Furthermore, the current finding of increased inferior frontal activation in relatives is consistent with research, suggesting that schizophrenia patients may use a different network in order to maintain task performance (Tan et al, 2006). Therefore, the decrease in DLPFC activity in relatives does not seem likely due to problems detecting brain activation in relatives.

In contrast to DLPFC dysfunction, relatives in this study did not exhibit ACC dysfunction. This suggests that ACC dysfunction may not be associated with the genetic liability for schizophrenia, but instead may be a consequence of the disorder. Therefore, ACC dysfunction could potentially be used as an illness marker and to help identify high-risk patients whose illness will likely convert to schizophrenia (Snitz et al, 2005). One issue for future research is to examine whether failure to detect decreased ACC activity in relatives could be due to PFC dysfunction in relatives. For example, PFC context processing is thought to increase processing of task-relevant stimuli, thereby reducing conflict. Therefore, if controls exhibited as little DLPFC activity as relatives, perhaps then controls would exhibit greater ACC activity than relatives. However, decreased ACC activity has been found in people with schizophrenia even when they fail to activate the DLPFC (Kerns et al, 2005; Snitz et al, 2005). Therefore, it does not seem likely that failure to detect ACC dysfunction was due to PFC dysfunction in relatives.

The results of the present study are generally consistent with previous functional imaging research on people with schizophrenia and their relatives. For example, similar to the current study, previous studies examining schizophrenia patients have found decreased DLPFC activity (eg, MacDonald and Carter, 2003; Snitz et al, 2005). In addition, in a previous study involving the Stroop task, people with schizophrenia did not significantly activate the DLPFC (although with a small sample size, patients did not differ significantly from controls at a conservative threshold; Kerns et al, 2005). Furthermore, previous studies with relatives of people with schizophrenia have found evidence of PFC dysfunction (Callicott et al, 2003a; Thermenos et al, 2004). Moreover, consistent with the results of the current event-related study, one other recent event-related imaging study found evidence of reduced left DLPFC activity in relatives (MacDonald et al, 2006). Hence, it appears that when preparing a response to specific cognitive control demands that relatives exhibit decreased DLPFC activity.

In addition, the finding of intact ACC activity in the current study is consistent with some previous research with relatives of people with schizophrenia (Callicott et al, 2003a; Thermenos et al, 2004). However, one previous study has reported reduced ACC activity in relatives (Whalley et al, 2004). However, one problem in interpreting ACC activity in these previous studies is that they involved tasks that do not necessarily strongly activate the ACC. In contrast, the current study involved a task that is known to activate the ACC in control participants (Carter et al, 2000), finding intact ACC activity in relatives.

Given evidence of DLPFC dysfunction both in people with schizophrenia and in their first-degree relatives, the current results suggest that DLPFC activity but not ACC activity may be impaired in relatives of individuals with schizophrenia. One issue for future research is to continue to 
examine possible specific genes that might be associated with DLPFC dysfunction in relatives of people with schizophrenia (Goldberg and Weinberger, 2004). Another issue for future research is to examine whether specific features of schizophrenia might be associated with DLPFC dysfunction in relatives of patients. For example, there is evidence that relatives of people with schizophrenia exhibit increased levels of disorganized speech (Docherty et al, 2004). Given that DLPFC dysfunction is associated with disorganization symptoms in people with schizophrenia (MacDonald et al, 2005), it is possible that disorganization symptoms in relatives of people with schizophrenia might reflect DLPFC dysfunction (eg, Kerns, 2006).

\section{ACKNOWLEDGEMENTS}

We thank Melissa Johnson and Ryan Walter for their help with running the study as well as the staff of the UPMC Magnetic Resonance Research Center for their help with data collection. This study was supported by National Institutes of Health (NIH) Grants MH059883 and MH066629. Data from this study were presented at the Society for Neuroscience Annual Meeting, San Diego, CA, October, 2004.

\section{DISCLOSURE/CONFLICT OF INTEREST}

Theresa Becker, Dr John Kerns, Dr Angus MacDonald, and Dr Cameron Carter reported no financial interests or potential conflicts of interest.

\section{REFERENCES}

Barch DM (2005). The cognitive neuroscience of schizophrenia. In: Cannon T, Mineka S (eds). Annual Review of Clinical Psychology, vol. 1 American Psychological Association: Washington, DC, pp 321-353.

Barch DM (2006). Introduction to special theme issue. Schizophr Bull 32: 486-488.

Botvinick MM, Braver TS, Barch DM, Carter CS, Cohen JD (2001). Conflict monitoring and cognitive control. Psychol Rev 108: 624-652.

Callicott JH, Bertolino A, Mattay VS, Langheim FJ, Duyn J, Coppola $\mathrm{R}$ et al (2000). Physiological dysfunction of the dorsolateral prefrontal cortex in schizophrenia revisited. Cereb Cortex 10: 1078-1092.

Callicott JH, Egan MF, Mattay VS, Bertolino A, Bone AD, Verchinksi B et al (2003a). Abnormal fMRI response of the dorsolateral prefrontal cortex in cognitively intact siblings of patients with schizophrenia. Am J Psychiatry 160: 709-719.

Callicott JH, Mattay VS, Verchinski BA, Marenco S, Egan MF, Weinberger DR (2003b). Complexity of prefrontal cortical dysfunction in schizophrenia: more than up or down. Am J Psychiatry 160: 2209-2215.

Carter CS, MacDonald III AW, Botvinick MM, Ross LL, Stenger VA, Noll D et al (2000). Parsing executive processes: strategic vs evaluative functions of the anterior cingulate cortex. Proc Natl Acd Sci USA 97: 1813-1817.

Carter CS, MacDonald III AW, Ross LL, Stenger VA (2001). Anterior cingulate cortex activity and impaired self-monitoring of performance in patients with schizophrenia: an event related fMRI study. Am J Psychiatry 158: 1423-1428.

Cohen JD, Botvinick M, Carter CS (2000). Anterior cingulate and prefrontal cortex: who's in control? Nat Neurosci 3: 421-423.
Cohen JD, Servan-Schreiber D (1992). Context, cortex, and dopamine: a connectionist approach to behavior and biology in schizophrenia. Psychol Rev 99: 45-77.

Cox RW (1996). AFNI: software for analysis and visualization of functional magnetic resonance neuroimages. Comput and Biomed Res 29: 162-173.

Docherty NM, Gordinier SW, Hall MJ, Dombrowski ME (2004). Referential communication disturbances in the speech of nonschizophrenic siblings of schizophrenia patients. J Abnorm Psychol 113: 399-405.

Evans AC, Collins DL, Mills SR, Brown ED, Kelly RL, Peters TM (1993). 3D statistical neuroanatomical models from 305 MRI volumes. Proc IEEE Nucl Sci Symp Med Imag Conf 3: 1813-1817.

First MB, Spitzer RL, Gibbon M, Williams JBW (1996). SCID-I/P, Version 2.0. Biometrics Research Department, New York State Psychiatric Institute: New York.

Fissell K, Tseytlin E, Cunningham D, Iyer K, Carter CS, Schneider $\mathrm{W}$ et al (2002). Fiswidgets: a graphical computing environment for neuroimaging analysis. Neuroinformatics 1: 111-125.

Forman SD, Cohen JD, Fitzgerald M, Eddy WF, Mintun MA, Noll DC (1995). Improved assessment of significant activation in functional magnetic resonance imaging (fMRI): use of a clustersize threshold. Magn Reson Med 33: 636-647.

Goldberg TE, Weinberger DR (2004). Genes and the parsing of cognitive processes. Trends Cogn Sci 8: 325-335.

Gottesman II, Gould TD (2003). The endophenotype concept in psychiatry: etymology and strategic intentions. Am J Psychiatry 160: 636-645.

Kerns JG (2006). Schizotypy facets, cognitive control, and emotion. J Abnorm Psychol 115: 418-427.

Kerns JG, Cohen JD, MacDonald III AW, Cho RY, Stenger VA, Carter CS (2004). Anterior cingulate conflict monitoring and adjustments in control. Science 303: 1023-1026.

Kerns JG, Cohen JD, MacDonald III AW, Johnson MK, Stenger VA, Aizenstein $\mathrm{H}$ et al (2005). Decreased conflict- and error-related activity in the anterior cingulate cortex in subjects with schizophrenia. Am J Psychiatry 162: 1833-1839.

Keshevan MS, Diwadkar VA, Spencer SM, Harenski KA, Luna B, Sweeney JA (2002). A preliminary functional magnetic resonance imaging study in offspring of schizophrenic parents. Prog Neuropsychopharmacol Biol Psychiatry 26: 1143-1149.

MacDonald III AW, Becker TM, Carter CS (2006). Functional MRI study of cognitive control in the healthy relatives of schizophrenia patients. Biol Psychiatry 60: 1241-1249.

MacDonald III AW, Carter CS (2003). Event-related fMRI study of context processing in dorsolateral prefrontal cortex of patients with schizophrenia. J Abnorm Psychol 112: 689-697.

MacDonald III AW, Carter CS, Kerns JG, Ursu S, Barch DM, Holmes AJ et al (2005). Specificity of prefrontal dysfunction and context processing deficits to schizophrenia in nevermedicated patients with first-episode psychosis. Am J Psychiatry 162: 475-484.

Manoach DS (2003). Prefrontal cortex dysfunction during working memory performance in schizophrenia: reconciling discrepant findings. Schizophr Res 60: 285-298.

Mayr U, Awh E, Laurey P (2003). Conflict adaptation effects in the absence of executive control. Nat Neurosci 6: 450-452.

Miller EK, Cohen JD (2001). An integrative theory of prefrontal cortex function. Annu Rev Neurosci 24: 167-202.

Pfohl B, Stagl D, Zimmerman M (1982). The Structured Interview for DSM-III Personality Disorders (SID-P). University of Iowa Department of Psychiatry: Iowa City, IA.

Rougier NP, Noelle DC, Braver TS, Cohen JD, O’Reilly RC (2005). Prefrontal cortex and flexible cognitive control: rules without symbols. Proc Natl Acad Sci USA 102: 7338-7343.

Snitz BE, MacDonald III AW, Cohen JD, Cho RY, Becker TM, Carter CS (2005). Lateral and medial hypofrontality in firstepisode schizophrenia: functional activity in a medication-naïve 
state and effects of short-term atypical antipsychotic treatment. Am J Psychiatry 162: 2322-2329.

Spitzer RL, Williams JBW, Gibbon M, First MB (1990). Structured Clinical Interview for DSM-III-R-Non-patient Edition (SCID-NP, Version 1.0). American Psychiatric Press: Washington, DC.

Tan HY, Sust S, Buckholtz JW, Mattay VS, Meyer-Lindenberg A, Egan $M$ et al (2006). Dysfunctional prefrontal regional specialization and compensation in schizophrenia. Am J Psychiatry 163: 1969-1977.

Thermenos HW, Seidman LJ, Breiter H, Goldstein JM, Goodman JM, Poldrack R et al (2004). Functional magnetic resonance imaging during auditory verbal working memory in nonpsychotic relatives of persons with schizophrenia: a pilot study. Biol Psychiatry 55: 490-500.

Whalley HC, Simonottom E, Flett S, Marshall I, Ebmeier KP, Owens DGC et al (2004). fMRI correlates of state and trait effects in subjects at genetically enhanced risk of schizophrenia. Brain 127: $478-490$.

Woods RP, Grafton ST, Holmes CJ, Cherry SR, Mazziotta JC (1998). Automated image registration: I. General methods and intrasubject, intramodality validation. J Comput Assist Tomogr 22: 139-152. 